Esta obra está bajo una Licencia Creative Commons Atribución-NoComercial-Compartirlgual 4.0 Internacional

(c) (1) (8) ()

Los estudios animales en la literatura hispanoamericana contemporánea

Alejandro Lámbarry

DOI: https://doi.org/10.24215/16696581e240

Recibido: 21-06-2019 Aceptado: 18-11-2019

\title{
Los estudios animales en la literatura hispanoamericana contemporánea
}

\section{Animal Studies in Contemporary Hispanic Literature}

Alejandro Lámbarry: alambarry.ffy|@gmail.com

https://orcid.org/0000-0003-3299-5914

Benemérita Universidad Autónoma de Puebla (México)

Resumen

Este artículo tiene como objetivo presentar un esquema general de la aportación de la literatura hispanoamericana contemporánea en la discusión crítica de los estudios animales. Hemos decidido, por motivos metodológicos dividir las obras en tres grupos: la figura del animal 1) cuestiona la ideología antropocentrista, 2) critica su propio estatuto de subordinación, 3) es símbolo de trascendencia mística. Los textos que presentamos -y que he abordado en el 
transcurso de mis investigaciones sobre el tema del animal en la literatura- son de autores mexicanos y argentinos de finales del siglo XX y comienzos del XXI. Más allá de su particularidad regional, hemos seleccionado a estos autores y a sus obras por su aportación temática a la crítica de los estudios animales.

Palabras clave: estudios animals; literatura hispanoamericana contemporánea; ética animal; voz animal.

\section{Abstract}

This article's main objective is the presentation of the late Hispano American literary contribution to the field of Animal Studies. In search for clarity and order we have divided the texts in four groups. Those that use the animal figure as 1) a means to question an anthropocentric ideology; 2) someone who critics the unequal relationship between human and animal, 3) a symbol of mystical transcendence. The authors that we have selected -and that I have analyzed and studied during the years-come from Mexico and Argentina and have written their works at the end of the XXth. and the beginning of the XXst. centuries. Our main goal is to study, regardless of their specific context, their contribution to the field of Animal Studies.

Keywords: animal studies; contemporary Hispanoamerican literature; animal ethics; animal voice.

Existe en los estudios animales una discusión sobre la posibilidad de que algún día sepamos con certeza lo que piensa, ve y siente otro animal que no seamos nosotros. Para el filósofo y científico Thomas Nagel tal posibilidad está fuera de toda cuestión. Nuestra subjetividad se halla siempre condicionada por nuestra manera de sentir y de pensar; imposible escapar de nuestro punto de vista. "I want to know what it is like for a bat to be a bat. Yet if I try to imagine this, I am restricted to the resources of my own mind, and those resources are inadequate to the task" (439).

Hay posturas al parecer opuestas a la de Nagel como, por ejemplo, la del autor sudafricano J.M. Coetzee, o, mejor dicho, de su narradora Elizabeth Costello, para quien(es) la imaginación empática es una herramienta sin límites: "Si con mi pensamiento puedo formarme un camino al interior de un ser que nunca ha existido, entonces puedo formarme un mismo camino al interior 
de un murciélago o un chimpancé o una ostra, todo ser con el cual comparto el sustrato de la vida" (35). El asunto aquí es que Coetzee no contradice a Nagel, más bien lo confirma, y nos pide a la vez que creamos en lo que el científico rechaza.

Un argumento en verdad contrario al de Nagel es el de Jacob von Uexküll, precursor de la etología. De acuerdo con él, la única manera de tener certeza y objetividad sobre un animal no humano es conocer el funcionamiento de sus sentidos, la manera en que interactúan con su cerebro y su mundo circundante. A esta actividad física y mental la llama Umwelt:

Podemos imaginarnos a todos los animales que conforman la naturaleza a nuestro alrededor (...) como encerrados dentro de una burbuja translúcida que circunscribe su espacio visual, y dentro de la cual está encerrado todo aquello que es visible al sujeto. Cada burbuja alberga otros lugares y en cada una se encuentra la dimensión y el espacio que confieren una estructura sólida a dicho espacio. (40)

Al comienzo de su libro Mondes animaux et monde humain, -la traducción al francés del libro Streifzüge durch die Umwellen von Tieren und Menschen: Bedeutungslehre- Von Uexküll da como ejemplo de Umwelt a la garrapata, que puede vivir inmóvil durante años a la espera del olor del ácido butírico (imperceptible para el humano), el cual le permitirá reproducirse y morir en un solo día. La etología es, por tanto, la ciencia que estudia la realidad desde el punto de vista de los animales; conocemos gracias a ella lo que ven, sienten, podrían pensar e imaginar un perro, las hormigas, los bonobos, etc. Sin embargo, como comenta Boris Cyrulnik (etólogo cognitivo), ahí donde termina la ciencia empiezan las artes. "Los artistas están ahí para expresar aquello que no escuchamos ni vemos en los animales: la alteridad portadora de sentido, la subjetividad, la singularidad, los misterios de sus miradas y de sus silencios al lado de los hombres" (203). ¿Cuáles han sido las respuestas de la literatura frente al animal? ¿De qué manera el animal ha impactado en nuestro imaginario cultural, afectivo y discursivo?

Podemos marcar dos momentos clave en la teoría literaria al momento de relacionarse con los estudios animales. El primero se ubica en Francia, con el pensamiento que desarrollaron Gilles Deleuze y Jacques Derrida desde el postestructuralismo. Los franceses cuestionaron la certeza discursiva del modernismo/humanismo y promovieron un pensamiento basado en conceptos performáticos que se definen en la frontera o en el devenir. El animal dejó de ser el reverso negativo del humano, aquel que obedecía a sus instintos, y se convirtió, en cambio, en un compañero en la búsqueda de una triada nueva de ética, epistemología y estética. Conceptos como "devenir animal", "animote", "jauría", "animal anómalo" son ejemplos de este pensamiento que se expresó con una mezcla de argumentación filosófica cuando refutaba, y de poesía o lenguaje literario cuando construía. El segundo momento clave ocurrió cuando la academia anglosajona adoptó la teoría francesa agregándole una agenda política. La teoría de los 
Estudios Animales nació en 2009 -año de la publicación de un número especial en Modern Language Review, la revista de la Modern Language Association (MLA). Esta agenda política de la academia anglosajona busca dar respuesta a lo que en Deleuze y Derrida se sugiere como una metáfora o una imagen poética, esto es: la formulación de sociedades de convivencia con los animales más justas y equitativas, junto con sus posibles derechos -como pacientes en lugar de agentes morales- y la construcción de identidades híbridas (o ciborg). Esta agenda se articula a través del producto cultural: televisión, cine y texto literario. (1)

Estos dos momentos teóricos resumen lo que las obras literarias expresaron en la narrativa latinoamericana, sobre todo a mediados del siglo XX y comienzos del XXI. Más allá de regionalismos y del contexto sociocultural de cada uno (Argentina y México) los/as autores/as que presentaremos en este artículo entran en el campo de los estudios animales en virtud de sus aportaciones a la discusión del tema animal. Para mayor claridad los hemos dividido en tres grupos, en los que la figura del animal: 1) cuestiona la ideología antropocentrista, 2) critica su propio estatuto de subordinación, 3) es símbolo de trascendencia mística. (2) El primer y el tercer grupo dialogan sobre todo con el pensamiento de Deleuze y Derrida, mientras que el segundo es un claro resultado de los Estudios animales adaptados en la escuela anglosajona.

1) El animal cuestiona la ideología antropocentrista

A mediados del siglo XIX surgió en la literatura anglosajona un grupo de textos que denunciaban la explotación y el maltrato animal. Nos referimos, por ejemplo, a la novela Black Beauty: The Autobiography of a Horse de Anna Sewell y a algunos cuentos de Mark Twain donde se narran las desgracias y abusos de los caballos de carreras que son sacrificados en la vejez o de perros que son usados como experimentos científicos. En estos textos los protagonistas animales generan empatía mediante la lástima, promueven un cambio apelando a la piedad. A pesar de su despertar crítico, la ideología cristiana que en gran medida provocó la subyugación animal se mantiene inamovible en estos textos. (3) Esto cambiará con la literatura de los autores argentinos César Aira y Griselda Gambaro, quienes buscan relativizar la ideología que subyace a la relación de poder desigual entre humanos y animales.

En el cuento "El hornero" de César Aira el pájaro del cono sur describe su entorno usando como contrapunto al humano. Para él, el lenguaje de los hombres era "funcional, simple, manejable; lo del hornero, el canto, el pío, era un garabato onírico en el que se mezclaban caóticamente la función y lo gratuito, el sentido y el sinsentido, la verdad y la belleza" (147). La vida del hornero es azarosa e impredecible, mientras que la del humano es lineal y obedece a un patrón muy claro, a su instinto. Los horneros son, en primer lugar, individuos, mientras que

Question, Vol. 1, N. ${ }^{\circ}$ 64, octubre-diciembre 2019. ISSN 1669-6581

Instituto de Investigaciones en Comunicación | Facultad de Periodismo y Comunicación Social | Universidad Nacional de La Plata La Plata | Buenos Aires | Argentina

Página 4 de 12 
el humano es ante todo una especie. Con Aira, el animal adopta de manera espontánea el discurso que subyace a su represión y abuso; es un discurso que al crear dicotomías reprime inevitablemente al otro; en este caso, lo hace mediante conceptos como instinto, especie, lenguaje sin sentido. La originalidad de Aira consiste en que revierte estos conceptos del animal, en quien normalmente son usados, al humano; relativiza así la estructura dual del discurso y avanza un paso -inevitable- para su posible reformulación.

En el cuento de Griselda Gambaro "Perro 1" la relación entre perro y dueño es conflictiva desde un inicio. El perro no cumple con el estereotipo de fidelidad canina: no recibe a su amo moviendo la cola, no le lame la mano, no muestra ninguna señal de aprecio. Debido a esto, la relación se va descomponiendo. "Me engañó la larga historia -fidelidad, abnegación- que él traía a sus espaldas" (95). El perro decide salir de la casa, pero después de algunos meses regresa golpeado y con hambre. Intentando ser gracioso, el perro adopta en cambio una actitud servil. "¿De qué servía, me pregunté, tener un perro que no se abalanzara cordialmente sobre uno? Que no agradecía ni comida, alfombra, ni correa" (98). La etóloga Alexandra Horowitz escribió que algunos científicos tenían la mala costumbre de generalizar el comportamiento de un animal en el de toda su especie; no obstante, jamás dejaríamos que el comportamiento de una sola persona representara a toda la especie humana. El cuento de Gambaro critica esta misma premisa. Sin una idea compleja del animal que rebase los estereotipos, estamos ejerciendo una acción de poder y discriminación sobre ellos. Los perros no son siempre fieles así como los hombres no comparten siempre una misma cualidad o defecto.

De acuerdo con lo anterior, este primer grupo se ubica en el umbral de la teoría postestructuralista, la cual cuestiona los grandes discursos por su carácter represivo de las diferencias y su notable antropocentrismo. Esto, con el fin de proponer lo que críticos como Milagros Ezquerro han Ilamado "minirrelatos": "historias que explican prácticas limitadas, acontecimientos locales y no conceptos globales o universales" (5). Son estos "minirrelatos" los que se expresan, de hecho, en el siguiente grupo.

2) El animal critica su propio estatuto de subordinación

La tradición del animal satírico en lengua española se remonta al Siglo de Oro. Y si tomamos otras tradiciones y lenguas, podemos llegar más atrás, hasta la épica animal del medievo (Roman le Renart) o las fábulas clásicas. En todos estos casos, el animal sirve para atacar los sistemas despóticos de su momento. Algo novedoso del siglo XX es que la figura del animal se utiliza por primera vez para criticar la relación de poder y subordinación en la que él es víctima, 
con el fin de crear nuevas relaciones de poder entre humanos y animales, además de sociedades más justas. Esto es posible gracias a la aportación teórica de los estudios animales.

En la literatura latinoamericana tenemos el caso de la novela La soledad de los animales del mexicano Daniel Rodríguez Barrón. En ella nos presenta una sociedad de capitalismo exacerbado, donde los animales se matan y se consumen como mercancía masiva. Esto genera entre los trabajadores de los rastros una gran desensibilización al sufrimiento y a la muerte. Laura, activista defensora de los derechos animales, organiza varias actividades para denunciar estos hechos y crear conciencia entre la población acerca de lo que sucede en los rastros. Para su desgracia, trabajadores de un rastro la sorprenden a medianoche en las premisas de la fábrica, la violan y la matan con una crueldad terrible: "abrieron sus cuerpos en canal y los rellenaron con pollo aún sin desplumar, tal vez incluso las aves estaban vivas pero atadas de las patas. Con su sangre escribieron en las paredes: "Todos somos animales" (84). Nínive, hija de Laura, decide continuar la lucha de su madre. Pero ya no caerá en el mismo error que ella. Para la niña, "lo primero que hay que hacer es no rescatar a los animales sino matarlos a todos... Sólo así dejarán de sufrir y sólo así, al menos por un momento, a sus captores se les acabará su negocio" (97). La novela termina cuando ella y Felipe disparan contra los animales encerrados en un retén del aeropuerto en la Ciudad de México.

La novela de Barrón actualiza el diálogo de los estudios animales desde la agenda política. El mercado de consumo de la carne no se puede adaptar fácilmente a una ética consciente de la diversidad natural. Es necesario abordar este problema desde una perspectiva compleja que comprende temas de economía, de política y de cultura. Las protestas con performances deben estar acompañadas de cambios en las dietas, en el funcionamiento de las fábricas y en las leyes.

Este conflicto ético se presenta en el cuento "Pájaros en la boca" de la autora argentina Samanta Schweblin. Una adolescente adquiere el hábito de comer pájaros vivos. La madre busca inútilmente una solución al problema por lo que decide dejar a la niña bajo el cuidado del padre. Surge entonces un proceso de animalización de la niña quien es referida a partir de pronombres o artículos y no de su nombre personal.

Cuando entraba a la casa, alrededor de las siete, y la veía tal cual la había imaginado durante todo el día, se me erizaban los pelos de la nuca y me daban ganas de salir y dejarla encerrada dentro con llave, herméticamente encerrada, como esos insectos que se cazan de chico y se guardan en frascos de vidrio hasta que el aire se acaba (cursivas nuestras) (Schweblin 85).

Question, Vol. 1, N. ${ }^{\circ}$ 64, octubre-diciembre 2019. ISSN 1669-6581

Instituto de Investigaciones en Comunicación | Facultad de Periodismo y Comunicación Social | Universidad Nacional de La Plata La Plata | Buenos Aires | Argentina

Página 6 de 12 
La adolescente se convierte poco a poco en animal, en cosa, en un enigma ante los ojos de quienes comen carne pero de otro tipo. La ingesta de caninos, de equinos y de cerdos puede resultar repulsiva a muchos pueblos que establecen con ellos relaciones de empatía o de repulsión sagrada. Las dietas obedecen, sin embargo, a normas culturales convencionales y arbitrarias. El hecho de que las asumamos como naturales revela su funcionamiento ideológico, que nada tiene que ver con lo racional. En el cuento de Schweblin, se nos revela la arbitrariedad con la que establecemos los criterios de selección de aquello que es correcto comer y cómo hacerlo. (Sin tocar aquí el tema de la producción, que se desarrolla con toda la brutalidad que puede imprimir el poder tecnológico actual al servicio de la acumulación monopólica global). (6)

3) El animal como símbolo de trascendencia mística

Una vez cuestionada y erradicada la frontera entre los seres animales y los seres humanos mediante la crítica de los "grandes discursos" de la modernidad y su antropocentrismo inherente, el animal presentó durante buena parte del siglo XX una duda existencial que impactó de varias maneras en la definición de lo que es -o debe ser- el humano y su relación con este otro radical. (4) Autores como el mexicano Leonardo da Jandra, en la segunda mitad del siglo XX, dirán que el animal es la trascendencia. Representa un estado de vida más pleno, en comunión con su entorno. Los animales no sufren la presión de sistemas ideológicos ni instituciones represivas; viven en una suerte de comunión con la realidad. Para ser congruente con su pensamiento, Da Jandra erradicó el estado de derecho y las relaciones de poder establecidas por éste; construyó una cabaña en la zona costera del estado mexicano de Oaxaca y aprendió de nuevo a ser independiente cazando su propio alimento. "La primera vez que maté un venado no pude evitar echarme de rodillas ante su cuerpo y ungirme con su sangre. Fue entonces cuando tuve el único vislumbre en mi vida de lo que podría ser la presencia de la divinidad" (14).

Seguir el impulso hacia la vida animal, entendida ésta como una vida carente de instituciones y de organización social compleja: esta propuesta fue también la del autor inglés D.H. Lawrence, quien, curiosamente, vivió una temporada en México. (5) Otra opción -menos radical- es buscar la animalidad en el interior, en la psicología de cada individuo humano. Esta opción está narrada en la novela de la mexicana Daniela Tarazona, El animal sobre la piedra. La protagonista de esta novela deja, sin explicación alguna, su casa y su ciudad para mudarse a la playa. Todos los días se recuesta sobre la arena y mira fijamente al mar. No hace otra cosa, 
hasta el momento en que siente una transformación en su cuerpo. Le brotan escamas en la piel, sus párpados se vuelven transparentes como los de los reptiles, su visión deja de dividir y catalogar sus percepciones: "No hay en los objetos un comienzo y un final, se encuentran unidos sin que pueda definir uno sin otro" (132). Escucha y entiende una voz que habla en un lenguaje no articulado: la voz "no era sonora, se manifestaba de modo peculiar, mediante zumbidos que no formaban medias palabras o siquiera vocales. Sin embargo, yo entendía" (153). La protagonista se convierte en un animal, en este caso, un ofidio. Tal metamorfosis no le genera angustia ni preocupación, por el contrario, logra una paz espiritual de tipo panteísta: se funde con su entorno.

El animal, en este caso, se encuentra inmerso en un discurso místico: es el intermediario entre el mundo divino y el terrenal; una puerta a una trascendencia ya no grupal, como en los sacrificios de la antigüedad, sino individual. (7) Su peligro radica en que, al cuestionar las dicotomías (razón/instinto, humano/animal), inevitablemente las revierte. Es decir, el animal y el instinto se convierten en el lado positivo de la balanza, pero la balanza permanece. Al presentar de manera más o menos estructurada este pensamiento, autores como Deleuze o Derrida no cometieron este error lógico, pero se mantuvieron siempre en suspenso, en el límite de una afirmación.

Devenir animal es precisamente realizar el movimiento, trazar la línea de fuga en toda su positividad, cruzar un umbral, alcanzar un continuo de intensidades que no valen más que por ellas mismas, encontrar un mundo de intensidades puras donde todas las formas se deshacen, también todas las significaciones, significantes y significados, en beneficio de una materia sin forma, de fluídos desterritolizadores, de signos asignificantes. (24)

Los franceses evitaron a toda costa inclinar la balanza hacia una u otra certeza, sin poder a su vez erradicarla con una solución racional. La ciencia, mediante la etología, ha corrido en este sentido con mayor suerte: ha erradicado la dicotomía con un universo plural donde conviven varios puntos de vista animales (el ser humano incluido). (8)

En los textos que aquí presentamos de autores argentinos y mexicanos la figura del animal renueva la tradición satírica -surgida en lengua española desde Cervantes con El coloquio de los perros- con los temas que impactan en nuestra época. En ellos se cuestiona el esquema que atribuye el lugar central al ser humano y se mira hacia otro en donde se promueve la convivencia verdadera, desde la epistemología, la ética y la estética. Algunos autores presentan este nuevo ser como un híbrido, y al animal como el factor de trascendencia. En otros casos, el interés se enfoca más en la ética y, por tanto, en lo social, con textos que plantean maneras de relacionarse con los animales desde una nueva ética, distinta tanto de la 
del capitalismo feroz como de la del humanismo liberal. Por último, subyace a ambos textos la crítica antropocentrista de los grandes discursos, que durante siglos representó el statu quo de la represión y la victimización del otro -ya fuera colonizado, mujer o, como en este caso, animal.

Empezamos este artículo con una discusión en el campo de la ciencia y nos gustaría terminar con este mismo tema. Es significativo que en América Latina no haya una tradición fuerte de textos literarios que, utilizando la etología, recreen la voz y/o el personaje animal. (9)

La ciencia nos explica ahora los grandes relatos; el de la creación del universo, los animales y la Tierra; nos explica la manera en que pensamos, imaginamos y sentimos. A diferencia de las religiones, la ciencia no pide que creamos ciegamente, nos da pruebas. Pero la ciencia no nos cuenta los grandes relatos. Para ello es necesario un narrador. Quizá nunca lleguemos a pensar y sentir con certeza lo mismo que piensan y sienten los animales, pero por primera vez podemos imaginarlos de una manera más científica, es decir, más veraz, con mayor certeza. EI animal ha representado en la literatura hispanoamericana un enigma existencial, una voz satírica que nos interpela como sociedad y en nuestra relación con el mundo circundante. Nos queda algo pendiente: relatar el mundo desde su punto de vista.

El escritor inglés E.M. Forster decía, a comienzos del siglo XX, que los personajes animales eran caricaturas, símbolos, mesas móviles o papeles pintados, nunca animales. Esto, sin embargo, podía cambiar:

The gulf that separates Man Friday from Batouala may be paralleled by the gulf that will separate Kipling's wolves from their literary descendants two hundred years hence... It is one of the ways where science may enlarge the novel, by giving it fresh subject-matter. (54)

No tuvimos que esperar doscientos años. La ciencia nos ha dado ya acceso al mundo desde la perspectiva animal. Es momento de que los escritores den el siguiente paso.

Notas

(1) En esta línea tenemos también una cercanía con el pensamiento feminista, que sugiere una comparación entre el abuso físico y la cosificación de las mujeres y la violencia y el asesinato de animales: la mujer como un objeto para la satisfacción sexual del hombre y el animal como un objeto para la satisfacción del apetito. 
(2) Existen pocas clasificaciones del narrador animal, entre ellas destacan las de William Nelles, quien realiza una clasificación basándose en la teoría estructuralista de la focalización. Nelles divide entre narrador homodiegético y heterodiegético. Narradores homodiegéticos hay tres: 1) la voz del animal es indistinguible de la del humano (Ejemplo: "The Stowaway" de Julian Barnes); 2) la voz del animal se distingue de la humana por medio de la utilización de estereotipos animales (El coloquio de los perros de Cervantes); 3) las voces del ser animal y del ser humano se encuentran integradas mediante la creación de un narrador dual (canción de Big Bill Broonzy's "Horny Frog"). El narrador heterodiegético recrea, por su parte, la voz animal de manera objetiva y certera. Da como ejemplo a Jack London: "London brilliantly supplements features of the animal's mental perspective (focalization proper) with features of his physical perspective (...), as he emphasizes the dog's 'field of vision' and the height of objects relative to the level of the canine gaze. (...) London's development of his animal filters, while intrinsically interesting, also serves his theme that animal's beliefs are more rational than those of humans" (192).

(3) Es el problema que aborda Cary Wolfe al escribir: "Indeed, one of the hallmarks of humanism -and more specifically of the kind of humanism called liberalism - is precisely its penchant for the sort of 'pluralism' that extends the sphere of consideration (intellectual or ethical) to previously marginalized groups without in the least destabilizing or throwing into question the schema of the human who undertakes such pluralization" (568).

(4) Es importante hacer notar que no hacemos alusión a la ciencia, con su experimentación y argumentación racionales, que incide en la creación, por ejemplo, de la etología. Pero sí hacemos alusión a la lectura banalizada que de la ciencia hizo un cientificismo popular new age, reforzando los estereotipos y las ideologías conservadoras de la época; por ejemplo, las dicotomías cuerpo/alma, instinto/razón. En esta lectura, Descartes es el primero en zanjar la frontera entre ellos y nosotros. Según Descartes, "un árbol produce frutos como un reloj marca la hora, y como los animales hacen lo que hacen" (Fontenay 283).

(5) D.H Lawrence escribe en sus cartas personales: "Mi gran religión es creer en la sangre, en la carne... Nuestra mente puede equivocarse. Pero lo que la sangre adivina, cree y dice es siempre la verdad" (59). De igual manera, bajo la influencia del misticismo oriental, Octavio Paz describe en El mono gramático el ser desde las cosas en su interioridad: "Las cosas nos revelan, sin revelar nada y por su simple estar ahí frente a nosotros, el vacío de los nombres, la falta de mesura del mundo, su mudez esencial" (Paz 100). En ambos casos, se trata de un vacío cercano a la trascendencia, una manera de expresar lo innombrable en lo salvaje, lo natural, lo animal. 
(6) Agradezco a Marissa Gálvez Cuen su ayuda en el análisis del cuento de Samantha Schweblin y la novela de Daniel Rodríguez Barrón. Una versión más extensa de este análisis aparecerá en la revista Iberoamericana.

(7) Este animal tiene relación, de igual manera, con un discurso posmoderno reflejado en libros como los de Steve Baker, The Postmodern Animal, y de Kari Weil, Why animal studies now? La destrucción de las metanarraciones convierte a la posmodernidad en una zona de oportunidades, en maneras de repensar lo humano, imaginar nuevas comunidades. Para Baker, la figura animal ayuda a "sanar la experiencia destructiva fragmentaria del mundo contemporáneo" (25). Baker afirma que la práctica artística es "una manera de alcanzar lo animal, alcanzar la asignificación, llegar más allá del significado, mediante una 'barrida', un 'incendio', un 'devenir'” (138). De acuerdo con Weil, "el giro hacia los animales en el arte al igual que en la teoría es un intento de imaginar un conocimiento diferente al de los humanos y, de esta manera, agrandar o cambiar las posibilidades de aquello en lo que podemos pensar y lo que podemos hacer en el mundo" (Weil 13). Se trata del giro contralingüístico que busca ir más allá del lenguaje, la última frontera del pensamiento humano.

(8) Susan McHugh identifica de manera precisa el problema de este tipo de animal trascendental: "Positing animals as mechanisms of transcendence, whether through foundational human knowledges or prediscursive fellow feeling for other species, only exacerbates this problem. Whatever the motive, such approaches to species risk a dangerous endgame for animal agency, one that brings animals into conversation only by emptying out the textual operations of species being and species becoming... not to mention the embodiments in space and time that animal studies scholarship seeks to pinpoint" (490).

(9) En su trilogía Las hormigas, el escritor francés Bernard Werber relata una historia de batallas, conquistas e invasiones de una comunidad de hormigas. Werber renueva el género de la épica con un personaje jamás antes visto y nos permite un conocimiento más vasto de este insecto

\section{Obras citadas}

Aira, César. Relatos reunidos. Buenos Aires, Mondadori, 2013.

Baker, Stephen. The Postmodern Animal. Londres, Reaktion, 2000.

Cervantes, Miguel de. Novelas ejemplares II. Madrid, Cátedra Letras Hispánicas, 2002.

Coetzee, J.M. The lives of animals. Princeton, Princeton University Press, 1999.

Da Jandra, Leonardo. La gramática del tiempo. Oaxaca, Editorial Almadía, 2009. 
Deleuze, Gilles. Mille Plateaux. Capitalisme et Schizofrénie 2. París, Les Éditions de Minuit, 1980.

Derrida, Jacques. L'animal que donc je suis. París, Éditions Galilée, 2006.

Ezquerro, Milagros. "Crisis y posmodernismo", presentado en el "Coloquio internacional

'Crisis, Apocalipsis y resistencias'”, Ruan, Francia. Febrero 2008.

Fontenay de, Elisabeth. Le silence des bêtes. La philosophie à l'épreuve de l'animalité. París, Fayard, 1998.

Forster, E.M. Aspects of the novel. Londres, Penguin Books, 1982.

Gambaro, Griselda. Los animales salvajes. Buenos Aires, Grupo Editorial Norma, 2006.

Horowitz, Alexandra. Inside of a Dog. What Dogs See, Smell, and Know. Nueva York, Scribner, 2009.

Lámbarry, Alejandro. El otro radical. La voz animal en la literatura

hispanoamericana.Puebla, Universidad Iberoamericana, Puebla, 2015-

McHugh, S. "Literary Animal Agents". PMLA. 124.2 (2009): 487-495. Impreso.

Nagel, Thomas. "What is it like to be a bat?" The Philosophical Review. Vol. 83, No. 4 (octubre, 1974), pp. 435-450

Nelles, William. "Beyond the Bird's Eye: Animal Focalization." Narrative, Contemporary Narratology (2001): 188-194.

Paz, Octavio. El mono gramático. Barcelona, Editorial Seix Barral, 1974.

Rodríguez Barrón, Daniel. La soledad de los animales. México, La Cifra, 2014.

Schweblin, Samantha (2009): "Pájaros en la boca". Etiqueta negra: 82-87. Web.

Sewell, Anna. Black Beauty: The Autobiography of a Horse. Londres, Empire Books, 2011.

Tarazona, Daniela. El animal sobre la piedra. Oaxaca, Editorial Almadía, 2008.

Twain, Mark. Mark Twain's Book of Animals. Berkeley, University of California Press, 2010.

Uexküll, Jacob von. Mondes animaux et monde humain. Suivi de La théorie de la signification.

París, Éditions Denöel, 1965.

Weil, Kari. Why animal studies now?, Nueva York, Columbia University Press, 2012.

Werber, Bernard. Les fourmis. París, Éditions Albin Michel, 1991.

Wolfe, Cary. "Human, all too human: 'Animal Studies' and the Humanities". PMLA.

(2009): 564-575. 